PSICOLOGIA

IBEROAMERICANA
Psicología Iberoamericana ISSN: 1405-0943

revista.psicologia@ibero.mx

Universidad Iberoamericana, Ciudad de México México

\title{
Vivencias de ansiedad por la pandemia por covid-19 en llamadas de urgencias psicológicas
}

Sánchez-Loyo, Luis Miguel; Morfín-López, Teresita; Vega-Michel, Claudia

Vivencias de ansiedad por la pandemia por covid-19 en llamadas de urgencias psicológicas

Psicología Iberoamericana, vol. 29, núm. 3, Esp., e293340, 2021

Universidad Iberoamericana, Ciudad de México, México

Disponible en: https://www.redalyc. org/articulo.oa?id=133968747010

DOI: https://doi.org/10.48102/pi.v29i3.340

\section{(c) (1)}

Esta obra está bajo una Licencia Creative Commons Atribución 4.0 Internacional. 
Artículos

\section{Vivencias de ansiedad por la pandemia por covid-19 en llamadas de urgencias psicológicas}

Experiences due to anxiety amid the COVID-19 epidemic in psychological emergency calls

Luis Miguel Sánchez-Loyo luis.sloyo@academicos.udg.mx

Psicología Iberoamericana, vol. 29, núm. 3, Esp., e293340, 2021

Universidad Iberoamericana, Ciudad de México, México

Recepción: 08 Febrero 2021 Aprobación: 05 Julio 2021

DOI: https://doi.org/10.48102/ pi.v29i3.340

Redalyc: https://www.redalyc.org/ articulo.oa?id $=133968747010$

\author{
Universidad de Guadalajara, México \\ https://orcid.org/0000-0001-8800-2622 \\ Teresita Morfín-López teremor@iteso.mx \\ ITESO - Universidad Jesuita de Guadalajara, México \\ (D) https://orcid.org/0000-0003-3994-3025 \\ Claudia Vega-Michel clavemi@iteso.mx \\ ITESO - Universidad Jesuita de Guadalajara, México \\ (D) https://orcid.org/0000-0002-8986-4459
}

Resumen: La pandemia de COVID-19 ha vulnerado la salud mental de las personas, pero se desconoce sus vivencias. El objetivo fue identificar las vivencias por ansiedad ante la pandemia de COVID-19 de usuarios de un servicio telefónico de urgencias psicológicas. Se realizó un estudio cualitativo retrospectivo exploratorio. Se seleccionaron 20 llamadas de urgencias psicológicas en Jalisco, México (21 usuarios, 16 mujeres), recibidas durante julio y agosto de 2020, con motivo de ansiedad asociada a la pandemia de COVID-19. Se realizó un análisis temático de las llamadas. Se identificaron vivencias de miedo y angustia; mayor ansiedad por incertidumbre ante padecer la enfermedad, contagiar a otros o que sus seres queridos se contagiaran; las formas para afrontar la pandemia fueron el distanciamiento social, nuevos hábitos de higiene, la modificación de sus rutinas de autocuidado y el consumo de información sobre la enfermedad. Los usuarios narraron dificultades con los profesionales de la salud para la atención a su malestar físico o mental.

Palabras clave: COVID-19, psicología cultural, vivencias, usuarios de urgencias psicológicas, ansiedad.

Abstract: The COVID-19 epidemic has affected people's mental health, but the emotions and the anxiety-related experiences caused by the epidemic are still unknown. The aim of the study was to identify the experiences of anxiety related to the COVID-19 pandemic amongst users of a psychological helpline. An exploratory retrospective qualitative study of psychological helpline incoming calls in Jalisco, Mexico was conducted. Twenty calls were selected ( 21 users, 16 women). These calls were recorded during July and August 2020 and were related to participants' feelings of anxiety during the COVID-19 epidemic. A thematic analysis of the users' narratives was carried out. Themes such as fear and anguish as relevant emotions; increased anxiety due to uncertainty about COVID-19 suffering; infecting others or the fear their loved ones would be infected; strategies to cope with the epidemic such as isolation, social distancing, new hygiene habits, the modification of their self-care routines and obtaining information about the disease, were found. Users reported difficulties with health professionals to care for their physical or mental discomfort since it interacts with various psychosocial vulnerabilities of the users.

Keywords: COVID-19, cultural psychology, experience, helpline users, anxiety. 


\section{Introducción}

La pandemia de COVID-19 ha provocado un menoscabo en la salud mental de las personas por el miedo a contraer la enfermedad, el confinamiento, la crisis económica asociada, entre otros factores. En población general en diversos países, se ha reportado mayor presencia de síntomas de depresión, ansiedad, mayor estrés (Hossain et al., 2020; Xiong et al., 2020) y menor bienestar subjetivo (Vindegaard \& Benros, 2020). En personas que han padecido COVID-19 se ha reportado mayores síntomas de depresión, de ansiedad, de estrés postraumático (Hossain et al., 2020; Vindegaard \& Benros, 2020), alteraciones del sueño (Hossain et al., 2020). En conjunto, los estudios han aportado evidencia de la afectación en la salud mental durante la pandemia.

En México, los efectos negativos de la pandemia de COVID-19 en la salud mental se han identificado. En población general mediante encuestas en línea con muestreos no probabilísticos con adultos de diferentes regiones de México, en las etapas iniciales de la pandemia, se identificó la presencia de síntomas de ansiedad (21\%) y depresión (27\%) (Galindo-Vázquez et al., 2020). En este mismo sentido, Priego-Parra y colaboradores (2020) identificaron una prevalencia de depresión de $28 \%$ y ansiedad de $50 \%$ en el mismo periodo de tiempo en adultos de diferentes regiones del país.

Algunas de las explicaciones en torno a los efectos negativos en la salud mental de la pandemia de COVID-19 han sido los factores de riesgo individuales y la política pública para afrontar la pandemia. Entre los factores de riesgo individuales sociodemográficos se han reportado ser mujer (Vindegaard \& Benros, 2020; Xiong et al., 2020), ser menor de 40 años, estar desempleado (Xiong et al., 2020) y trabajar en servicios de salud (Rajkumar, 2020). Entre los factores de riesgo individuales relacionados con la salud se identificaron antecedentes de trastorno mental (Xiong et al., 2020), condiciones de salud frágiles (Vindegaard \& Benros, 2020), estar enfermo o con síntomas compatibles con COVID-19 (Rajkumar, 2020). Entre otros factores de riesgo se identificó la exposición frecuente a información sobre la pandemia (Xiong et al., 2020), estar en confinamiento forzoso (Lei et al., 2020; Tang et al., 2021) y tener parientes enfermos con COVID-19 (Vindegaard \& Benros, 2020). Un metanálisis realizado por Santabárbara y colaboradores (2021) identificó, además de los ya mencionados, tener un empleo precario, problemas financieros, preocupación por estar enfermo o enfermar a sus seres queridos, consumo abusivo de alcohol y tabaco. Este mismo estudio señaló que los mayores niveles de ansiedad fueron en los periodos de más casos identificados de COVID-19 (Santabárbara et al., 2021). En México, se ha identificado que ser mujer, soltero, no tener hijos, padecer una enfermedad médica preexistente, antecedentes de malestar emocional (Galindo-Vázquez et al., 2020), ser joven, con problemas de sueño y presentar adicción al internet (Priego-Parra et al., 2020) incrementaron la probabilidad de presentar ansiedad y depresión. 
Respecto de los factores de riesgo asociados a las políticas públicas para afrontar la pandemia, han sido el confinamiento y el distanciamiento social (Rajkumar, 2020; Vindegaard \& Benros, 2020), desinformación, falta de certidumbre e incapacidad para predecir la evolución de la pandemia (Rajkumar, 2020).

Los servicios de atención psicológica vía telefónica han sido un recurso para hacer frente a la pandemia. Estos servicios han reportado los diversos problemas de salud mental en sus usuarios. En España, 76\% de los usuarios referían sintomatología ansiosa y depresiva, $21 \%$ preocupación o miedo por padecer COVID-19, 20\% conflictos familiares (Berdullas Saunders et al., 2020). En Suiza, identificaron un incremento en las llamadas de personas mayores de 65 años (29\%), los problemas reportados fueron miedo a ser infectado, soledad y lidiar con el día a día (Brülhart $\&$ Lalive, 2020). Otro estudio en Grecia identificó que $84 \%$ de las llamadas estaban relacionadas con el confinamiento, 56\% incapacidad para descansar y $53 \%$ soledad; más aún, $20 \%$ fueron identificados con síntomas de ansiedad, 37\% con depresión y 23\% crisis de pánico (Peppou et al., 2020). En México, la Línea de la Vida de los Centros de Integración Juvenil entre mayo y agosto de 2020, identificaron en el $68 \%$ de los usuarios problemas de estrés y ansiedad, 15\% depresión, 31\% problemas económicos, 33\% con síntomas de COVID-19, 57\% haberse contagiado o temer haberse contagiado; $50 \%$ mencionaron estar intranquilos y $16 \%$ sentirse rebasados por las dificultades (Díaz-Negrete et al., 2020).

Más allá de los datos cuantitativos, el contenido de las llamadas telefónicas recibidas por los servicios de atención psicológica podría ser de utilidad para una mayor comprensión de las vivencias de las personas durante la pandemia de COVID-19. Sin embargo, los estudios son limitados. En Bangladesh, se reportaron ansiedad y falta de sueño en $87 \%$ de las llamadas, $42 \%$ con problemas de salud física o preocupación por los tratamientos o pruebas de COVID-19, 29\% reportaron dificultades para el manejo del tiempo durante el confinamiento, problemas por el trabajo de oficina en casa, falta de descanso, entre otros (Iqbal et al., 2020). Otro estudio en India (Joshi et al., 2020) identificó que las llamadas de los usuarios presentan varios motivos simultáneamente. Los motivos identificados fueron estar ansiosos por síntomas parecidos a COVID-19, por los riesgos de contagio por contacto con objetos y por los costos y el lugar para recibir atención médica (Joshi et al., 2020). Este mismo estudio señaló que los usuarios reportaron síntomas de ansiedad, depresión, soledad, sentirse sobrepasados o desmotivados, siendo más importantes en personas con antecedentes de problemas mentales (Joshi et al., 2020). Dichos estudios hacen referencia a aspectos mayormente individuales, identificados de manera aislada, dejando de lado aspectos contextuales y culturales en interacción con los individuos.

Por lo anterior, este estudio se aborda desde una perspectiva psicocultural, es decir, desde una perspectiva de relación entre los procesos psicológicos producto de la interacción de individuos entre individuos y de individuos con su medio ambiente, en un intento de superar las dicotomías entre individuo/sociedad y pensamiento/emoción (Subero 
\& Esteban-Guitart, 2020; Veresov, 2017). En esta línea, Vygotski hace una propuesta hacia una noción de consciencia como sistema psicológico complejo integrando las funciones afectivas y cognitivas, destacando la relación del sujeto con el mundo de una forma activa, en el proceso de desarrollo humano (Vygotski, 1993, 1994, 1996, 2007). Esta propuesta de Vygotsky (1993) señala que la psique se podría comprender como "neoformaciones" o sistemas dinámicos en los cuales la emoción y el pensamiento interactúan dinámicamente (Del Río \& Álvarez, 2007).

En la psicología cultural, los sentidos subjetivos se definen como unidades simbólico-emocionales que emergen ante la presencia de otros o de determinados eventos y experiencias, sin que esos eventos sean su causa directa (González Rey, 2011; González Rey \& Mitjáns Martínez, 2017). Es decir, estos sentidos subjetivos generan una realidad psicológica, social y cultural, que a su vez permite generar nuevos sentidos subjetivos, en un proceso recursivo, dinámico y activo (González Rey, 2017; Goulart, 2019). Esta subjetividad no sigue una lógica racional, sino que se configura a partir de diversas experiencias-vivencias y se expresa y desarrolla de una manera particular a lo largo de la vida. La subjetividad social se considera como un flujo permanente de acciones y procesos que tienen lugar en los diferentes espacios sociales de la actividad humana, donde aparecen diferenciadas y sintetizadas las configuraciones subjetivas de las distintas personas, prácticas y escenarios de vida y actividad (González Rey, 2017).

Lo psicológico en este planteamiento no se limita a elementos individuales o contextuales separados, sino a través de una lógica que propone enmarcar eventos particulares en el tiempo y el espacio, en los cuales la emoción y la cognición se encuentran articulados en acciones y procesos subjetivos dinámicos, las cuales hay que observar y analizar para poder comprender la conducta humana. Desde esta perspectiva la vivencia / perezhivanie cubre e integra como una unidad dinámica, la totalidad del individuo que actúa y se ve afectado/transformado por y en el entorno (Subero \& Esteban-Guitart, 2020).

Por lo que la pregunta de investigación a responder fue ¿cuáles son las vivencias por ansiedad ante la pandemia de COVID-19 expresadas en usuarios de un servicio telefónico de urgencias psicológicas en México?

\section{Método}

Para la elaboración de este estudio, partimos de la perspectiva teórica de la psicología cultural expuesta previamente. El objetivo del estudio fue identificar las vivencias por ansiedad ante la pandemia de COVID-19 de usuarios de un servicio telefónico de urgencias psicológicas en México. Se pretende identificar las vivencias, las emociones, acciones y procesos subjetivos de las personas, en relación con su entorno inmediato y mediato, en el tiempo y espacio del confinamiento.

El diseño del estudio es de tipo cualitativo retrospectivo exploratorio. Para ello, se recabaron 20 llamadas y en ellas participaron 21 personas (en una llamada participaron dos usuarios) de un servicio de atención psicológica de urgencias vía telefónica en Jalisco. Las llamadas fueron 
recibidas durante julio y agosto de 2020. En esos meses, Jalisco se mantuvo en naranja en el semáforo de riesgo epidémico con limitaciones importantes a la vida social.

El servicio de urgencias psicológicas por teléfono fue organizado por parte del Gobierno de Estado de Jalisco para apoyar a la población para hacer frente a la pandemia de COVID-19. Funciona las 24 horas y es atendido por profesionales de la psicología capacitados en intervención en crisis. El servicio de urgencias psicológicas está vinculado con otras líneas de servicios de urgencias estatales como 911 y línea de atención a la emergencia por COVID-19 (servicio para la detección y canalización de personas con posibles síntomas de COVID-19). Las características sociodemográficas de las personas que habían solicitado el apoyo en el servicio de urgencias psicológicas al corte de agosto de 2020 fueron mujeres en proporción 2 a 1.5 respecto de hombres, entre los 25 a los 45 años, con estudios de licenciatura o bachillerato, los estados civiles más frecuentes fueron solteros y casados, en proporción semejante.

Antes de determinar los criterios de selección de las llamadas se realizó una exploración aleatoria del contenido y duración de las llamadas para identificar las características más adecuadas y así responder la pregunta de investigación. Las llamadas fueron seleccionadas de manera retrospectiva bajo los siguientes criterios: duración mayor de ocho minutos, usuarios identificados como viviendo en el Estado de Jalisco, en la codificación de la llamada realizada por los psicólogos operadores deberían aparecer las palabras: COVID o confinamiento y ansiedad o angustia.

Las llamadas seleccionadas tuvieron una duración promedio de 22 minutos (rango de 8 a 70 minutos). Las llamadas fueron transcritas textualmente y fueron analizadas las narraciones hechas por los usuarios durante las mismas. Las intervenciones psicológicas no fueron analizadas. Cabe señalar que uno de los autores participó como psicólogo operador voluntario en el servicio (ninguna de sus llamadas resultó seleccionada), el resto de los autores no participaron como psicólogos operadores.

El contenido relevante fue analizado mediante un análisis temático de acuerdo con Braun y Clarke (2006). Éste implicó que los autores se familiarizaran con el contenido de las llamadas, generando inicialmente códigos relacionados con la experiencia subjetiva del participante. Estos códigos fueron organizados en temas y subtemas, adjuntando los extractos relevantes de las llamadas, relacionados con estos códigos (Braun \& Clarke, 2006). Los temas y subtemas se organizaron con base en el objetivo del estudio e inductivamente con base en el contenido de las llamadas. Se fueron comparando entre los investigadores los resultados de este análisis, a fin de ir llegando a acuerdos sobre los códigos y categorías del procedimiento analítico. El análisis temático fue realizado a nivel semántico con base en significados identificados durante la revisión de las transcripciones.

El contenido codificado de las llamadas permitió identificar una categoría general: la vivencia, que comprende las siguientes subcategorías: a)emociones durante la pandemia b)formas de afrontar la pandemia c)incertidumbre ante el diagnóstico y los tratamientos $\mathrm{d}$ )presencia de un 
trastorno mental e)condiciones de vulnerabilidad psicosocial: mala salud física, precariedad económica, desempleo y vivir solo.

\section{Consideraciones éticas}

Al iniciar la llamada se presenta un menú de opciones en el cual se incluyen las condiciones del servicio prestado. Se hace referencia expresa a que los datos recabados podrían ser usados, salvaguardando la identidad de los usuarios. Todos los datos de identificación de los usuarios fueron omitidos en las transcripciones de las llamadas. El aviso de privacidad está disponible para todos los usuarios y fue aprobado por el comité de ética del Instituto Jalisciense de Salud Mental. El presente estudio se dio en el marco de un convenio de colaboración entre instituciones educativas y del gobierno estatal promovido para la implementación de la estrategia de prevención y contención de la salud mental en el Estado de Jalisco.

\section{Hallazgos}

Es importante situar los hallazgos de este estudio en el marco de la vivencia, así como de la subjetividad, individual y social. Las experiencias de los participantes en este estudio, relacionadas con la pandemia y el confinamiento, configuran vivencias que requieren ser comprendidas como eventos cognitivo-afectivos dinámicos, en los no puede reducirse la explicación psicológica a elementos individuales o contextuales, por separado. Sino que, a partir de una lógica transaccional, enmarcarse en eventos particulares a partir de los cuales entender la conducta humana (Subero \& Esteban-Guitart, 2020).

En las expresiones de los usuarios podemos identificar que en torno a la pandemia por COVID-19 y el confinamiento, los usuarios tienen vivencias similares, que permiten reconocer una problemática social que se refiere como individual, y que es recuperada a través de la comunicación que se establece con los psicólogos operadores que atienden las llamadas telefónicas.

El diálogo entre el usuario y el psicólogo operador refiere a eventos particulares que hacen posible identificar la relación entre contexto e individuo, cognición y emoción, en un flujo constante y dinámico. Es posible reconocer la subjetividad en las acciones y los procesos narrados (González Rey et al., 2019).

\section{Características de los usuarios}

Respecto de las características de los usuarios se identificó su sexo, su rango de edad, con quienes vivían al momento de hacer la llamada y contacto con personas enfermas por COVID-19 (véase Tabla 1). Los usuarios fueron 21 personas ( 16 mujeres), todos mayores de edad, 15 de ellos eran adultos, tres jóvenes menores de 30 años, tres mayores de 60 años. Respecto de con quienes convivían: tres personas vivían solas, tres vivían con su pareja, 
cinco vivían con su pareja e hijos, tres vivían con sus padres, seis vivían con otros familiares como hermanos, abuelos, hijos y nietos, un usuario vivía con su hijo menor. Cinco usuarios habían enfermado o estaban enfermos por COVID-19, 3 tenían un familiar o vecino con COVID-19, 13 usuarios no habían conocido personalmente a alguien con COVID-19. Cabe señalar que en una llamada participaron dos usuarios, uno después del otro.

Tabla 1

Tabla 1

Características sociodemográficas de los usuarios.

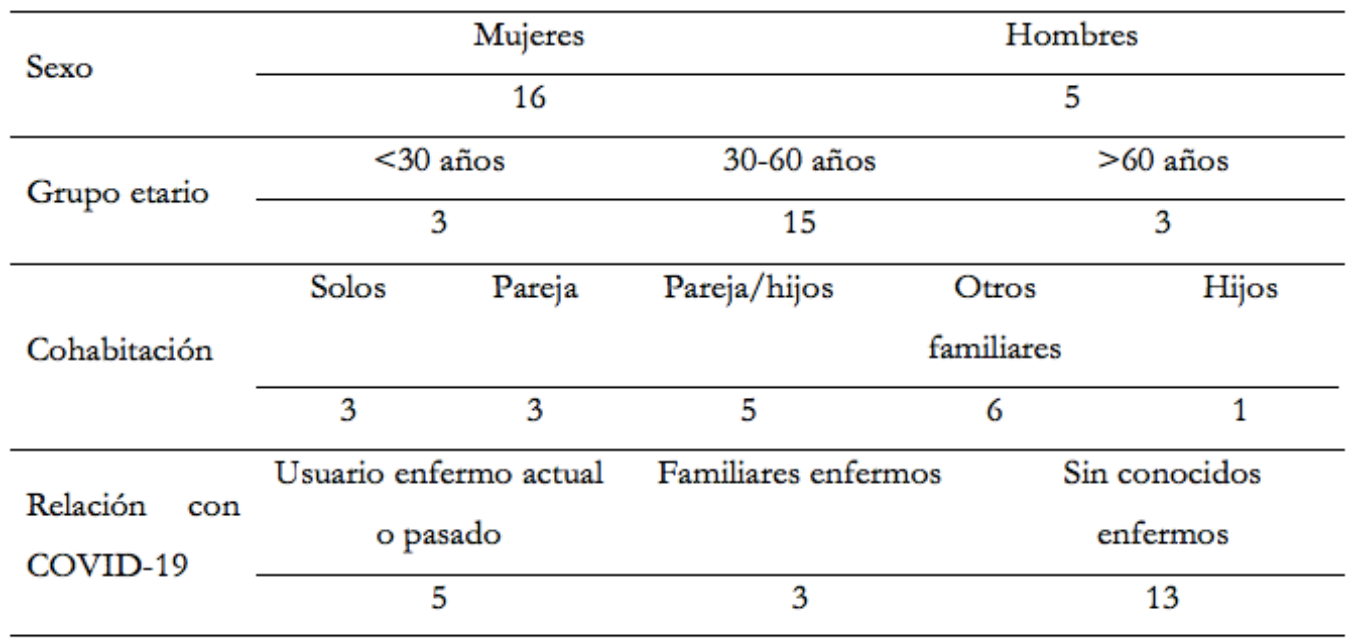

\section{Vivencias}

Como señalamos anteriormente el objetivo de este estudio fue identificar las vivencias por ansiedad ante la pandemia de COVID-19 en usuarios de una línea telefónica de urgencias psicológicas en México.

La vivencia se entiende como: la manera en cómo las personas valoran, perciben e interpretan aquello que les sucede y les rodea desde su contexto sociocultural (Esteban Guitart, 2008). La categoría vivencia en este estudio está configurada por cinco subcategorías, que permiten organizar algunos de los elementos de la vivencia de ansiedad en el confinamiento por COVID-19. Estas subcategorías emergieron inductivamente durante el análisis.

Estas subcategorías son las siguientes:

a) Emociones durante la pandemia.

b) Formas de afrontar la pandemia.

c) Incertidumbre ante el diagnóstico y los tratamientos.

d) Presencia de un trastorno mental.

e) Vulnerabilidad psicosocial: salud física, precariedad económica, desempleo y vivir solo. 
Se presenta a continuación las subcategorías y las expresiones de los participantes que hacen referencia a las mismas.

\section{a) Emociones durante la pandemia}

Se entienden las emociones como las evaluaciones positivas o negativas de un suceso pasado, presente o futuro vinculadas a procesos cognitivos, relacionadas con consideraciones sociales (Rodríguez Salazar, 2008). Respecto de las emociones reportadas por los usuarios, la emoción más reportada fue el miedo, expresado en diversas maneras como: angustia, preocupación, pavor, temor. Los usuarios mencionaban miedo relacionado a enfermar por COVID-19, ellos o sus seres queridos, o si ya estaban enfermos, contagiar a sus seres queridos.

"Yo tengo tanto miedo de tocar las cosas que sean de la calle (...) me dice [un familiar] que haga el esfuerzo para que no me paralice el miedo"

(Participante 18, mujer adulta entre 35 a 39 años)

"Estoy asustada. Tengo mucho miedo (...), porque al otro lado de mi casa hay un [lugar] y dos personas salieron positivas"

(Participante 9, mujer adulta mayor de 60 años)

"...le tengo mucho miedo al coronavirus, pienso que me va a dar a mi COVID y que me voy a morir"

(Participante 15, hombre adulto entre 40 y 44 años)

"...tengo mucho miedo, me duele el alma, a mi [familiar] le pegó COVID" (Participante 16, mujer adulta entre 35 y 39 años)

Como se puede observar en el participante 15 , el miedo a enfermar por COVID-19 estaba asociado a la muerte propia, o en la participante 16 con la enfermedad de un ser querido.

Otra emoción reportada por los usuarios fue nerviosismo, estar estresado, desesperado o con ansiedad, esto igualmente por el riesgo de enfermar de COVID-19, ya fuera el usuario o sus seres queridos.

"Ando bien nerviosa porque, a la mañana salí, yo casi no salgo (...) yo no he salido sin cubrebocas (...) porque yo no me quiero enfermar, porque tampoco quiero enfermar a mi [familiar]"

(Participante 3, mujer adulta entre 45 y 49 años)

“...sigue mi ansiedad, porque ahora pienso que la persona que vino pudo traer el virus (...) estaba preocupado, si se limpió bien, es un tema ya como de paranoia"

(Participante 2, hombre adulto entre 40 y 44 años)

El nerviosismo se ve vinculado a la incertidumbre de la eficacia de las medidas de protección al entrar en interacción con otras personas. Ambos usuarios expresaron que tomaban medidas para reducir el riesgo de contagio, pero con el riesgo latente de contraer la enfermedad.

\section{b) Formas de afrontar la pandemia}

Se entiende el afrontamiento como un proceso social en el cual las personas evalúan el problema entendiéndolo como individual o compartido, así como las acciones individuales o colectivas para afrontarlo (Lyons et al., 1998). Los usuarios refieren como una forma de hacer frente a la pandemia seguir las recomendaciones dadas por las autoridades como el uso de cubrebocas, limpieza en sus personas y en casa, mantener el distanciamiento social. 
"He estado aislado, hemos estado haciendo el aislamiento correcto." (Participante 2, hombre adulto entre 40 y 44 años)

"yo casi no salgo (...) yo no he salido sin cubrebocas...porque yo no me quiero enfermar (...) me agarró del hombro, y yo pienso que ya me contaminó (...) [al regresar a casa] le eché agua con jabón y vinagre, lo lavé y lo tendí (...) la sana distancia es muy difícil de tomarla a los metros que le dicen a uno [en un mercado].”

(Participante 3, mujer adulta entre 45 y 49 años)

“...no salgo de mi cuarto (...), cuando salgo al baño voy con cubrebocas, no les hablo [a quienes viven con él], lo menos que se pueda contacto." (Participante 10, hombre joven entre 25 y 29 años)

Las expresiones de los usuarios dan muestra de ser conscientes y de seguir puntualmente las recomendaciones para reducir el contagio emitidas por las autoridades como se observa en la participante tres. En este mismo sentido, el participante dos refiere hacer el aislamiento "correcto".

Otra forma de afrontar es buscar información sobre la enfermedad, los síntomas, las medidas de protección, aunque los usuarios reconocen es excesiva y les genera malestar emocional como se observa en los participantes 2 y 12 .

"...me estoy perdiendo mucho en las noticias, eso me afecta y me provoca problemas."

(Participante 2, hombre adulto entre 40 y 44 años)

“...me he metido a muchas páginas y en una te dicen unas cosas y en otra te dicen que ya hay nuevos síntomas (...) es lo que me está angustiando mucho" (Participante 12, mujer adulta entre 40 y 44 años)

“...llegó un momento en que todos los días estaba revisando cuantos casos se habían acumulado y hace unos días una de mis [familiares] ya me dijo que evitara de ver tantas noticias"

(Participante 18, mujer adulta entre 35 y 39 años)

Ambas formas para afrontar la pandemia les pueden llevar a tener más miedo o ansiedad; ya que, por un lado, no es posible saber si las medidas de higiene y distancia social son eficaces para evitar enfermar de COVID-19, Por otro lado, la información a la que recurren puede ser contradictoria, imprecisa o alarmista. Ambas acciones de afrontamiento a la epidemia se realizan como actos individuales y no de manera colectiva.

c) Incertidumbre ante el diagnóstico de su padecimiento y los tratamientos a seguir

Los usuarios mencionaron acudir a las instituciones de salud para recibir diagnóstico y tratamiento adecuado, pero no tuvieron el resultado deseado provocando una vivencia de incertidumbre. Los usuarios dan cuenta de las limitaciones institucionales y del sector de salud para dar el diagnóstico y el tratamiento esperado ante la pandemia.

En aquellos usuarios enfermos o preocupados por estar enfermos por COVID-19, privaba la incertidumbre ante su diagnóstico o su tratamiento médico. La vivencia de incertidumbre es más clara cuando han sido tratados a distancia. Esto podría ser una fuente de miedo y preocupación para los usuarios por el temor a morir, enfermar gravemente 
o enfermar a otros. También, es claro que los usuarios afrontan la posible presencia de síntomas de COVID-19, atendiendo las recomendaciones del personal sanitario, pero ello no limita la incertidumbre como se puede observar en las siguientes expresiones.

"Cuando me dieron la cita para COVID, solo me dijeron: toma paracetamol para que se te baje la fiebre y en caso de tener dificultad para respirar ve a tu clínica."

(Participante 21, mujer joven entre 25 y 29 años)

"...solo me hice la prueba, me hablaron de Línea COVID, me dijeron que si no tenía signos de alarma que no era necesario [ir a revisión]. En persona no he visto a un médico."

(Participante 13, mujer adulta entre 35 y 39 años)

“...ayer fui a la farmacia (...), porque me sentía muy desesperada, la doctora me checó, me dijo que estaba bien, que era ansiedad (...) lo que sí me preocupó fue que me dijo que, si seguía con esto, que fuera a urgencias, sentí que se contradijo."

(Participante 12, mujer adulta entre 40 y 44 años)

Las personas con probables crisis de ansiedad viven con mayor incertidumbre, ya que los problemas respiratorios sentidos por ellos podrían ser síntomas de COVID-19 o síntomas de ansiedad, cada uno de ellos con distintos tratamientos. Los participantes 12 y 21 dan ejemplos claros de dicha incertidumbre y sus interacciones con la ansiedad.

“...me dijo la persona que me atendió [en Línea COVID] que más probablemente sea crisis de ansiedad que tengo."

(Participante 12, mujer adulta entre 40 y 44 años)

“...ayer empecé otra vez con los mismos síntomas de cuando tenía coronavirus, lo mismo que sentía, no sé si relacionarlo con la ansiedad, una recaída o que todavía no me recupero [de COVID-19] (...) Tengo trastorno de ansiedad y como me dan ansiedades mis síntomas antes del COVID, era falta de aire."

(Participante 21, mujer joven entre 25 y 29 años)

Los usuarios dan cuenta del problema de la incertidumbre del diagnóstico y la posibilidad de padecer algo grave. Los usuarios afrontan la incertidumbre consultando a más médicos, realizándose distintos estudios y tomando diversos medicamentos, lo que no llevó a una certeza sobre su diagnóstico.

“...empecé hace [número de días] con los síntomas de COVID (...) decidí ir con un doctor particular, me dio medicamento, me sentí un poquito mejor (...) cuando se terminó el medicamento, empezaron todos los síntomas fuertes de lo que fue el COVID (...) [pasados días] con la doctora me dijo que me iba a dar el mismo medicamento (...) me recomendaron un doctor, estudios, pensando que era [nombre de otra enfermedad]. Consulté otro doctor, finalmente el doctor me dice: ¿̇abes qué? (...) te vamos a hacer varios estudios para ver si no tienes [nombre de una tercera enfermedad]."

(Participante 10, hombre joven entre 25 y 29 años)

“...le hablé a la doctora que me trata (...) hace días empecé con hormigueo en la garganta (...) la doctora me dijo que yo presentaba una 
[nombre de enfermedad] , se me quitó (...) [días después] le hablo a la doctora y le digo que hoy amanecí con el [parte del cuerpo] que me hormigueaba más (...), ya me dijo que me fuera a un hospital (...) voy con otra doctora, me examina, pero me dice que traigo el COVID (...) Me dijo, es que tienes que hablar para que te hagan la prueba (...) ya hablé [a Línea COVID] y la persona que me contestó me dijo que no tengo los síntomas, que tengo que esperarme los siete días para volver a llamar y que me diagnostiquen porque no tengo los síntomas."

(Participante 11, mujer adulta entre 50 y 54 años)

Estos dos participantes dan muestra de esfuerzos personales y no institucionales por llegar a un diagnóstico sobre su malestar, pero se encuentran con ambigüedades e imprecisiones por el sector salud. La falta de un tratamiento claro y adecuado podría aumentar el miedo y la angustia por la posibilidad de la muerte o del contagio a sus seres queridos.

d) Presencia un trastorno mental

Se identificó en las narraciones de los usuarios la suposición, por parte del personal sanitario que les atendió, de presentar un problema mental como ansiedad como se observa en las siguientes expresiones.

“...hablé a Línea COVID, pues porque me sentía a lo mejor con síntomas de la enfermedad [COVID-19], pero pues no, me dijo la persona que me atendió, que eran síntomas de ansiedad"

(Participante 12, mujer adulta entre 40 y 44 años)

“...me dice [el médico], estás muy estresada, traes una angustia muy fuerte, dijo y necesitas un psiquiatra."

(Participante 11, mujer adulta entre 40 y 44 años)

Algunos usuarios no tenían consciencia de padecer algún trastorno mental. El sugerirles un tipo de trastorno mental podría generar incertidumbre y miedo, al dejar de lado sus presuntos problemas de salud física motivo de sus consultas en el sector salud.

Otros usuarios refirieron antecedentes de atención en salud mental, pero solo conforme se desarrollaba la llamada o la pregunta directa del psicólogo operador. Una vez expresado esto, los usuarios abundan en su malestar como se observa en los siguientes participantes.

"...tengo mi ansiedad todo descontrolada, me siento mal ahorita, tengo así como [número] años en tratamiento [psiquiátrico] (...) tengo ansiedad y depresiones."

(Participante 15, hombre adulto entre 40 y 44 años)

"...yo estoy enferma de los nervios me han internado en [institución de salud mental] (...) soy límite de la personalidad con depresión.”

(Participante 16, mujer adulta entre 35 y 39 años)

\section{e) Vulnerabilidad psicosocial}

Las vivencias antes mencionadas: las emociones, la forma de afrontar la pandemia, la incertidumbre del diagnóstico y el tratamiento a recibir por sus síntomas, así como tener un posible trastorno mental aparecen en el contexto de condiciones de vulnerabilidad psicosocial como: precariedad económica, desempleo, problemas de salud física y vivir solo. Estas condiciones de vulnerabilidad psicosocial complejizan la manera de afrontar la pandemia, provocan mayor miedo y angustia ante la 
posibilidad de la enfermedad y limitan el acceso a diversos servicios de salud.

En las narraciones fue posible identificar precariedad económica en los usuarios de manera que afecta la manera de afrontar la pandemia, como en los siguientes participantes:

"No tengo dinero para hacerme otra prueba [de COVID-19], donde me alcanzaba era en [nombre de la institución] pero no tienen cupo."

(Participante 21, mujer joven entre 25 y 29 años)

“...luego la economía, están cerrando y cerrando, no hay trabajo y los que me ayudan se la están viendo difícil."

(Participante 3, mujer adulta entre 45 y 49 años)

“...por la situación económica y la escasez de empleo se nos obliga a permanecer dentro de [su área de trabajo] si es que queremos conservar el empleo (...) tengo un [familiar] que tiene [nombre de enfermedad crónica] (...) para mí es muy difícil lidiar con dicha situación porque necesito mucho mi trabajo."

(Participante 4, mujer adulta entre 40 y 44 años)

La presencia de desempleo apareció en las narraciones, pudiendo incrementar el estrés, y el miedo:

"Al principio me quedé sin empleo (...) tengo un niño de [número] años, y pues vivo con mis [familiares]."

(Participante 5, mujer joven entre 20 y 24 años)

En las narraciones se identificó como vulnerabilidad psicosocial la mala salud física en los usuarios con la presencia de enfermedades crónica, que en el contexto de la pandemia se ven relegadas por las instituciones de salud.

"Soy una persona diabética."

(Participante 11, mujer adulta entre 50 y 54 años)

"Yo soy hipertensa (...) me diagnosticaron un tumor (...), me iban a operar y ya no me pudieron operar [por la pandemia]."

(Participante 14, mujer adulta entre 45 y 49 años)

Se identificaron usuarios viviendo solos, su situación limita el acceso a recursos de apoyo familiar y comunitario.

"Vivo aquí (...) solo, (...) me dio neumonía atípica, no le avisé a mis [familiares] para no preocuparles."

(Participante 10, hombre joven entre 25 y 29 años)

\section{Discusión}

Sobre las características sociodemográficas de los usuarios se observan semejanzas con otros estudios. La mayoría de los usuarios fueron mujeres, entre los 30 y 60 años, 8 usuarios habían enfermado o tenido familiares enfermos por COVID. Los estudios sobre factores de riesgo para presentar ansiedad durante la pandemia de COVID-19 indican que las mujeres son un grupo vulnerable (Vindegaard \& Benros, 2020; Xiong et al., 2020). Respecto de otras características reportadas en estudios parecidos, los resultados son contradictorios. Respecto del sexo de los usuarios, el estudio realizado en la Línea de la Vida reporta igual número 
de mujeres y hombres (Díaz-Negrete et al., 2020) lo que pudiera deberse a ser servicio identificado con la atención a adicciones más prevalentes en hombres; en cambio el estudio en España $73 \%$ de los usuarios fueron mujeres (Berdullas Saunders et al., 2020). Sobre la edad de los usuarios se observan diferencias con un estudio suizo con mayor registro de de adultos mayores posiblemente asociado a la propia dinámica poblacional del país (Brülhart \& Lalive, 2020). En cambio en otro estudio español, los usuarios fueron adultos entre los 40 y 59 años (Berdullas Saunders et al., 2020) y otro estudio mexicano con media de 43 años (Díaz-Negrete et al., 2020) los que coinciden con el presente estudio. Es posible que los usuarios de los servicios de atención psicológica estén en relación, por un lado, con la población con mayor necesidad, pero asociado a la cobertura y conocimiento del servicio en la comunidad, por grupo etario o género.

Comprender las vivencias de los usuarios con relación a la ansiedad en el confinamiento por COVID-19 desde una perspectiva integral, permite reconocer ciertos padecimientos que se producen en contextos y condiciones determinadas. En nuestro estudio pudimos observar que aun cuando las llamadas se hacen en una conversación individual, en donde se expresa la subjetividad de la persona, al codificar y categorizar las expresiones, se puede reconocer una subjetividad social, que se produce al experimentar condiciones similares durante el confinamiento por COVID-19.

Sobre las vivencias de miedo y ansiedad ante el contagio de COVID han sido reportadas en trabajos cualitativos y cuantitativos. La ansiedad ha sido reportada como un problema relevante durante la pandemia (Galindo-Vázquez et al., 2020; Priego-Parra et al., 2020). Los sentimientos asociados a la pandemia en Argentina fueron temor, miedo, angustia (Johnson et al., 2020) al igual que en el presente estudio. El temor por estar enfermo o por contagiar a seres queridos fue reportado por otros estudios en servicios de atención psicológica telefónica (Díaz-Negrete et al., 2020; Iqbal et al., 2020; Joshi et al., 2020). La incertidumbre ante la evolución de la pandemia, la pérdida de la continuidad en la vida cotidiana, el aislamiento y el miedo a contraer la enfermedad se han asociado fuertemente a la ansiedad y el miedo (Johnson et al., 2020; Rajkumar, 2020; Santabárbara et al., 2021). Particularmente, la incertidumbre en la pandemia de COVID-19 se ha asociado a mayor presencia de síntomas de ansiedad y depresión (Del Valle et al., 2020). ¿Podemos considerar estos síntomas como exclusivamente individuales y separados del contexto de confinamiento y la pandemia?

La vulnerabilidad psicosocial de los usuarios ante la pandemia, aunado a las condiciones de distanciamiento social y la atención en salud ante síntomas semejantes de COVID-19, incrementaron el miedo y la ansiedad. Se identificaron personas solas o con escasa familia, empleos precarios, problemas de salud física y mental que dificultan la gestión de las emociones, los pensamientos y las acciones ligados al riesgo de contagio de COVID-19. Se considera que estas condiciones de vulnerabilidad colocan a estas personas en mayor riesgo de presentar ansiedad y depresión durante la pandemia de COVID-19 (Santabárbara et al., 2021; Vindegaard \& 
Benros, 2020; Xiong et al., 2020). De mayor vulnerabilidad son quienes presentan padecimientos mentales previos (Galindo-Vázquez et al., 2020; Joshi et al., 2020; Santabárbara et al., 2021). En el presente estudio, fueron notables las narraciones de personas que externaron su preocupación excesiva por enfermar de COVID-19, misma que fue considerada por profesionales de salud en la línea telefónica de identificación de casos de COVID-19 y por los psicólogos operadores del servicio de urgencias psicológicas telefónicas como signo de un posible trastorno de ansiedad. Sin embargo, para el usuario, el posible diagnóstico de trastorno de ansiedad no le permite controlar su miedo y angustia, ya que las sensaciones de dificultad respiratoria se confunden con los síntomas de COVID-19.

Las vivencias muestran el problema estructural del sistema de salud para lidiar con personas con vulnerabilidades múltiples. Los usuarios dan cuenta de derivaciones en el sector salud para cumplir con las demandas de atención. Los usuarios reportaron pasar de un médico a otro, de una institución o servicio a otro, incluso en servicios telefónicos para detección de casos de COVID-19 y los de urgencias psicológicas. Estas derivaciones aumentan la incertidumbre y podrían incrementar el miedo y la angustia de los usuarios, desgasta los servicios de salud, incrementando la sintomatología ansiosa y provocando un uso ineficiente de los recursos, debido a la búsqueda de certeza de un diagnóstico para sus síntomas. Las vivencias de miedo, angustia e incertidumbre interactúan con las características y el funcionamiento del sistema de salud durante la pandemia. Estas circunstancias se encuentran en relación dinámica con la manera en cómo las personas valoran, perciben e interpretan aquello que les sucede y les rodea desde su contexto sociocultural (Esteban Guitart, 2008).

Otra forma de afrontar la situación fue el consumo de noticias relacionadas con la pandemia. Incluso, los propios usuarios consideraron que dicho consumo abusivo de información favorecía la aparición de síntomas de ansiedad, por la incapacidad de poder determinar la certeza y veracidad de la información. Estos efectos nocivos en la salud mental por el consumo inmoderado de noticias relacionadas con la pandemia han sido reportados en diversos estudios (Priego-Parra et al., 2020; Rajkumar, 2020; Xiong et al., 2020).

Los usuarios mencionan hacer uso de recursos mayormente personales $\mathrm{y}$ atendiendo a una responsabilidad individual para afrontar la pandemia siguiendo las instrucciones de las autoridades de distanciamiento social, comunicándose a los servicios públicos vía telefónica, consultando profesionales de la salud, pero sin encontrar solución a su problemática. Fueron notables los esfuerzos referidos por apegarse a las medidas de distanciamiento social e higiene, este mismo hallazgo fue reportado por Johnson y colaboradores (2020). Se identificaron usuarios que no acudieron a visitar al médico a pesar de tener síntomas importantes de COVID-19, por seguir estrictamente las indicaciones recibidas, aquellos que cambiaron sus rutinas de supervisión de enfermedades crónicas por no acudir a las unidades médicas para sus revisiones, los cambios en los 
hábitos provocaron alteraciones importantes en la vida de las personas durante la pandemia.

Los usuarios reportan el agotamiento de sus recursos económicos y de apoyo familiar por la vulnerabilidad asociada a la pandemia. Las narraciones expresan dificultad económica para las pruebas de COVID-19 en instituciones privadas, para continuar con sus tratamientos sin asistir a instituciones públicas, pérdida del empleo apoyándose con familiares para la sobrevivencia, así como soportar abusos laborales por la necesidad de conservar el empleo. Particularmente, en estudios en países en vías de desarrollo como México, se ha reportado más preocupación por la situación económica que por enfermar de COVID-19 (Díaz-Negrete et al., 2020; Iqbal et al., 2020; Joshi et al., 2020).

\section{Conclusiones}

Se identificó que las vivencias de los participantes se relacionan con emociones de miedo y angustia; las formas de afrontar la pandemia fueron el aislamiento, el distanciamiento social, la incorporación de hábitos de higiene, la modificación de sus rutinas de autocuidado, el consumo de información sobre la enfermedad y la búsqueda de un diagnóstico para sus síntomas en el sistema sanitario. La incertidumbre ante el diagnóstico y el tratamiento se relaciona con temor a contagiar a otros, no tener claridad sobre si padecen COVID-19, confundir la sensación de falta de aire con la dificultad respiratoria al padecer COVID-19; el no tener la certeza del diagnóstico, lleva a consultar varios médicos, hacerse diversos estudios y tomar distintos medicamentos hasta el agotamiento de sus recursos. En relación con la presencia de un posible trastorno mental, los usuarios no identifican por si mismos un padecimiento mental, sino es el psicólogo operador o el médico quien sugiere al usuario tener una crisis o síntomas de ansiedad. En cuanto a las condiciones de vulnerabilidad psicosocial como: problemas de la salud física y mental, precariedad económica, desempleo y vivir solo, aumentan las vivencias de incertidumbre, las formas de afrontamiento desgastantes y el miedo y la angustia ante la posibilidad de enfermar de COVID-19.

El presente estudio recupera las vivencias en voz de los usuarios con ansiedad en la pandemia de COVID-19, lo que permite corroborar mediante datos cualitativos los hallazgos de estudios cualitativos ya reportados. Por otro lado, permite aportar al conocimiento de los servicios de atención vía telefónica de urgencias médicas, psicológicas o de asistencia por COVID-19, sobre la complejidad de las vivencias de los usuarios y la necesidad de una mejor integración de los servicios de salud, de forma que los usuarios no se consideren menospreciados o no atendidos. Los datos recabados podrían ayudar a una mejor comprensión por parte de los profesionales de salud, de las vivencias durante la pandemia y su articulación con emociones, contexto e interacciones de los usuarios, las interacciones con otras personas y el sistema de salud mismo. 
Las limitaciones del presente estudio son diversas. Por un lado, estriban en el limitado número de llamadas analizadas, analizar un mayor número de llamadas podría consolidar los hallazgos presentados en el presente estudio. Por otro lado, hay que reconocer que se tratan de llamadas recolectadas en un determinado tiempo (julio-agosto 2020) y espacio (Estado de Jalisco) del confinamiento de los usuarios durante la pandemia de COVID-19. Es necesario recolectar datos de otros servicios de ayuda psicológica telefónica en otras ubicaciones geográficas en México, así como en diferentes periodos de tiempo a lo largo de la pandemia vigente, para identificar las vivencias.

Los hallazgos podrían ayudar a diseñar políticas públicas para los usuarios con múltiples vulnerabilidades, que tienen vivencias de no encontrar la respuesta adecuada en el sistema de salud a sus problemáticas y las emociones asociadas a ello.

\section{Agradecimientos}

El presente estudio se realizó con apoyo de la Secretaria de Salud Jalisco y el Instituto Jalisciense de Salud Mental. El estudio no contó con financiamiento. Los autores declaran no tener conflicto de intereses.

\section{Referencias}

Berdullas Saunders, S., Gesteira Santos, C., Morán Rodríguez, N., Fernández Hermida, J. R., Santolaya, F., Sanz Fernández, J., \& García-Vera, M.P. (2020). El teléfono de asistencia psicológica por la covid-19 del ministerio de sanidad y del consejo general de la psicología de España: características y demanda. Revista Española de Salud Pública, 94, ei-13.

Braun, V., \& Clarke, V. (2006). Using thematic analysis in psychology. Qualitative Research in Psychology, 3(2), 77-101. https://doi.org/10.119 1/1478088706qp063oa

Brülhart, M., \& Lalive, R. (2020). Daily suffering: Helpline calls during the COVID-19 crisis. Covid Economics, 19, 143-158.

Del Río, P., \& Álvarez, A. (2007). From the psychology of drama to the drama of psychology: The relationship between the life and work of Lev S. Vygotsky. Estudios de Psicologia, 28(3), 303-332. https://doi.org/10.1174/0210939 07782506489

Del Valle, M. V., Andrés, M. L., Urquijo, S., Yerro-Avincetto, M., LópezMorales, H., \& Canet-Juric, L. (2020). Intolerance of uncertainty over COVID-19 pandemic and its effect on anxiety and depressive symptoms. Revista Interamericana de Psicologia/Interamerican Journal of Psychology, 54(2), e1335. https://doi.org/10.30849/ripijp.v54i2.1335

Díaz-Negrete, D. B., Rodríguez-Kuri, S. E., Cruz-Cortés, C. de J., GutiérrezLópez, A. D., González-Sánchez, J. D., \& Fernández-Cáceres, C. (2020). Problemática psicosocial y de salud mental reportada por consultantes del servicio de atención telefónica línea de la vida, derivados a Centros de Integración Juvenil durante la epidemia de COVID-19. Revista Internacional de Investigación en Adicciones, 6(2), 45-60. https://doi.org /10.28931/riiad.2020.2.06 
Esteban Guitart, M. E. (2008). Hacia una psicología cultural. Origen, desarrollo y perspectivas. Fundamentos en Humanidades, IX(18), 7-23.

Galindo-Vázquez, O., Ramírez-Orozco, M., Costas-Muñiz, R., MendozaContreras, L. A., Calderillo-Ruíz, G., \& Meneses-García, A. (2020). Síntomas de ansiedad, depresión y conductas de autocuidado durante la pandemia de COVID-19 en la población general. Gaceta de México, 156(4), 298-305 https://doi.org/10.24875/GMM.20000266

González Rey, F. (2011). A re-examination of defining moments in Vygotsky's work and their implications for his continuing legacy. Mind, Culture and Activity, 18(3), 257-275. https://doi.org/10.1080/10749030903338517.

González Rey, F. (2017). The topic of subjectivity in psychology: Contradictions, paths and new alternatives. Journal for the Theory of Social Behaviour, 47(4), 502-521. https://doi.org/10.1111/jtsb.12144

González Rey, F. L., \& Mitjáns Martínez, A. (2017). Subjetividade: teoría, epistemologia e método. Alinea

González Rey, F., Mitjáns Martínez, A., \& Goulart, D. M. (2019). The topic of subjectivity within cultural-historical approach: Where it has advanced from and where it is advancing to. En F. L. González Rey, A. Mitjáns Martínez \& D. Goulart (Eds.), Subjectivity within cultural-historical perspective: Theory, methodology and research (pp. 3-19). Springer.

Goulart, D. M. (2019). Subjectivity and life: In memory of Fernando González Rey. Mind, Culture and Activity, 26(2), 102-107. https://doi.org/10.108 0/10749039.2019.1619775

Hossain, M. M., Tasnim, S., Sultana, A., Faizah, F., Mazumder, H., Zou, L., McKyer, E. L. J., Ahmed, H. U., \& Ma, P. (2020). Epidemiology of mental health problems in COVID-19: A review. F1000Research, 9, 636. https:/ /doi.org/10.12688/f1000research.24457.1

Iqbal, Y., Jahan, R., Yesmin, S., Selim, A., \& Siddique, S. N. (2020). CoVID 19 - related issues on tele - counseling helpline in Bangladesh. AsiaPacific Psychiatry, e12407. https://doi.org/10.1111/appy.12407

Johnson, M. C., Saletti-Cuesta, L., \& Tumas, N. (2020). Emociones, preocupaciones y reflexiones frente a la pandemia del COVID-19 en Argentina. Ciência \& Saúde Coletiva, 25(suppl 1), 2447-2456. https://d oi.org/10.1590/1413-81232020256.1.10472020

Joshi, A., Tammana, S., Babre, T., \& Kallianpur, R. (2020). Psychosocial response to COVID - 19 pandemic in India: Helpline counsellors' experiences and perspectives. Counselling and Psychotherapy Research, 21(1), 19-30. https://doi.org/10.1002/capr.12378

Lei, L., Huang, X., Zhang, S., Yang, J., Yang, L., \& Xu, M. (2020). Comparison of prevalence and associated factors of anxiety and depression among people affected by versus people unaffected by quarantine during the COVID-19 epidemic in Southwestern China. Medical Science Monitor, 26, e924609-1-e924609-12. https://doi.org/10.12659/MSM.924609

Lyons, R. F., Mickelson, K. D., Sullivan, M. J., \& Coyne, J. C. (1998). Coping as a communal process. Journal of Social and Personal Relationships, 15(5), 579-605. https://doi.org/10.1177/0265407598155001

Peppou, L. E., Economou, M., Skali, T., \& Papageorgiou, C. (2020). From economic crisis to the COVID-19 pandemic crisis: Evidence from a mental health helpline in Greece. European Archives of Psychiatry and 
Clinical Neuroscience, 271, 407-409. https://doi.org/10.1007/s00406-02 0-01165-4

Priego-Parra, B. A., Triana-Romero, A., Pinto-Gálvez, S. M., Ramos, C. D., Salas-Nolasco, O., Reyes, M. M., Ramos-de-la-Medina, A., \& RemesTroche, J. M. (2020). Anxiety, depression, attitudes, and internet addiction during the initial phase of the 2019 coronavirus disease (COVID-19) epidemic: A cross-sectional study in México. medRxiv. 2020.05.10.20095844. https://doi.org/10.1101/2020.05.10.20095844

Rajkumar, R. P. (2020). COVID-19 and mental health: A review of the existing literature. Asian Journal of Psychiatry, 52, 102066. https://doi.org/10.10 16/j.ajp.2020.102066

Rodríguez Salazar, T. (2008). El valor de las emociones para el análisis cultural. Papers: Revista de Sociología, 87, 145-159. http://dx.doi.org/10.5565/rev /papers/v87n0.793.

Santabárbara, J., Lasheras, I., Lipnicki, D. M., Bueno-Notivol, J., Pérez-Moreno, M., López-Antón, R., De la Cámara, C., Lobo, A., \& Gracia-García, P. (2021). Prevalence of anxiety in the COVID-19 pandemic: An updated meta-analysis of community-based studies. Progress in NeuroPsychopharmacology and Biological Psychiatry, 109, 110207. https://doi.o rg/10.1016/j.pnpbp.2020.110207

Subero, D., \& Esteban-Guitart, M. (2020). Hacia una psicología de la experiencia. Aportaciones de la psicología transaccional y la teoría de la subjetividad. Revista Puertorriqueña de Psicologia, 31(1), 20-35.

Tang, F., Liang, J., Zhang, H., Kelifa, M. M., He, Q., \& Wang, P. (2021). COVID-19 related depression and anxiety among quarantined respondents. Psychology \& Health, 36(2), 164-178. https://doi.org/10.1 080/08870446.2020.1782410

Veresov, N. (2017). The concept of Perezhivanie in cultural-historical theory: Content and contexts. En M. Flee, F. González Rey \& N. Veresov (Eds.), Perezhivanie, emotions and Subjectivity (pp. 47-70). Springer. https://doi .org/10.1007/978-981-10-4534-9_3

Vindegaard, N., \& Benros, M. E. (2020). COVID-19 pandemic and mental health consequences: Systematic review of the current evidence. Brain, Behavior, and Immunity, 89, 531-542. https://doi.org/10.1016/j.bbi.20 20.05.048

Vygotski, L. S. (1993). Pensamiento y lenguaje. En L. S. Vygotski (Ed.), Obras escogidas, tomo II (pp. 9-348). Aprendizaje Visor.

Vygotski, L. S. (1994). The problem of the environment. En R. van der Veer \& J. Valsiner (Eds.), The Vygotsky reader (pp. 338-355). Blackwell.

Vygotski, L. S. (1996). El problema de la edad. En L. S. Vygotski (Ed.), Obras escogidas, tomo IV (pp. 251-273). Aprendizaje Visor.

Vygotski, L. S. (2007). Psicología del arte. Fundación Infancia y Aprendizaje.

Xiong, J., Lipsitz, O., Nasri, F., Lui, L. M. W., Gill, H., Phan, L., Chen-Li, D., Iacobucci, M., Ho, R., Majeed, A., \& McIntyre, R. S. (2020). Impact of COVID-19 pandemic on mental health in the general population: A systematic review. Journal of Affective Disorders, 277, 55-64. https://doi. org/10.1016/j.jad.2020.08.001 